17. Makarenko A., Moskalkov M. U.S.S.R. Computational Mathematics and Mathematical Physics. Springer Translation. 1983. Vol. 23. 999 p.

\title{
NEW APPROACHES FOR REDUCING ARTIFICIAL OSCILLATIONS IN NUMERICAL SOLUTIONS. ANTI-DIFFUSION, ANTI-DISPERSION AND LONGOLIERS
}

Abstract. Two most known errors is the artificial smoothing of the solution and oscillations in the solutions near the places with high derivatives of the solutions (near the sharp fronts of the solution). Some methods of improving numerical solutions of evolution equations are proposed on the base of theoretical considerations. The artificial viscosity and artificial dispersion for difference schemes of gas dynamics are proposed as the first examples. A new class of tools for improving numerical solutions is proposed - «Langoliers». «Langoliers» are special difference operators which should be applied at each time steps after the running of original difference schemes. The design of «Langoliers» allows reducing the dissipative and dispersive errors of schemes. The examples are anti-diffusion, anti-dispersion and specially constructed difference schemes.

Key words: numerical schemes; dispersion; dissipation; non-smooth solutions, anti-dispersion; «Langoliers»; non-linear problems.

Получено 15.02.2019

УДК 517.946

DOI: $10.32626 / 2308-5878.2019-19.71-77$

В. В. Маринець, д-р фіз.-мат. наук,

О. І. Когутич, магістр

ДВНЗ «Ужгородський національний університет», м. Ужгород

\section{ПРО ОДИН ПІДХІД ДОСЛІДЖЕННЯ КРАЙОВОЇ ЗАДАЧІ ДЛЯ КВАЗІЛІНІЙНОГО РІВНЯННЯ ГІПЕРБОЛІЧНОГО ТИПУ 3 РОЗРИВНОЮ ПРАВОЮ ЧАСТИНОЮ}

Будується конструктивний швидкозбіжний двосторонній метод дослідження та наближеного розв'язання крайової задачі для квазілінійного хвильового рівняння на площині з розривною правою частиною в області із складною структурою краю. Встановлюються достатні умови існування функцій порівняння, регулярного або іррегулярного розв'язку розглядуваної крайової задачі, його єдиності та знакосталості.

Ключові слова: «вільні» криві, іррегулярний розв'язок, функиї порівняння, умови узгодження.

Вступ. Крайові задачі для квазілінійних рівнянь гіперболічного типу з неперервними правими частинами в різних областях із складною структурою краю розглядались в роботах [1-3], в яких встановлено до- 
статні умови існування та єдиності їх регулярних або іррегулярних розв'язків. Дана робота є продовженням досліджень, приведених у [4].

Розглянемо в $R^{2}$ область $D=D_{1} \cup D_{2} \cup D_{3}$ [5, с. 250], де

$$
\begin{gathered}
D_{1}=\left\{(x, y) \mid x \in\left(x_{0}, x_{1}\right], y \in\left(y_{0}, y_{1}\right]\right\}, \\
D_{2}=\left\{(x, y) \mid x \in\left[x_{0}, x_{1}\right], y \in\left(y_{1}, g_{1}(x)\right)\right\}, \\
D_{3}=\left\{(x, y) \mid x \in\left(x_{1}, x_{2}\right], y \in\left(g_{2}(x), y_{1}\right]\right\}, \\
x_{0}<x_{1}<x_{2}, \quad y_{0}<y_{1}<y_{2},
\end{gathered}
$$

a $y=g_{r}(x) \Leftrightarrow x=\kappa_{r}(y), r=1,2$ - «вільні» криві, причому $g_{r}^{\prime}(x)>0$, $g_{1}\left(x_{r-1}\right)=y_{r}, g_{2}\left(x_{r}\right)=y_{r-1}$.

Позначимо $D^{*}:=D \backslash E_{1} \cup E_{2}$,

де

$$
E_{1}=\left\{(x, y) \mid x \in\left[x_{0}, x_{1}\right], y=y_{1}\right\}, E_{2}=\left\{(x, y) \mid y \in\left[y_{0}, y_{1}\right], x=x_{1}\right\} .
$$

Досліджується наступна крайова задача: в просторі функцій $C^{*}(\bar{D}):=C^{(1.1)}\left(D^{*}\right) \cap C(\bar{D})$ знайти розв'язок диференціального рівняння

$$
\begin{gathered}
U_{x y}(x, y)+a_{1}(x, y) U_{x}(x, y)+a_{2}(x, y) U_{y}(x, y)= \\
=f(x, y, U(x, y)):=f[U(x, y)],
\end{gathered}
$$

який задовольняє крайові умови

$$
\begin{gathered}
U\left(x_{0}, y\right)=\psi(y), y \in\left[y_{0}, y_{1}\right], U\left(x, y_{0}\right)=\varphi(x), x \in\left[x_{0}, x_{1}\right], \\
U\left(x, g_{1}(x)\right)=\omega_{1}(x), x \in\left[x_{0}, x_{1}\right], \\
U\left(x, g_{2}(x)\right)=\omega_{2}(x), x \in\left[x_{1}, x_{2}\right], \\
\psi\left(y_{0}\right)=\varphi\left(x_{0}\right), \psi\left(y_{1}\right)=\omega_{1}\left(x_{0}\right), \varphi\left(x_{1}\right)=\omega_{2}\left(x_{1}\right),
\end{gathered}
$$

a функція $f[U(x, y)]=f_{s}\left[U_{s}(x, y)\right],(x, y) \in \overline{D_{s}}, \quad s=1,2,3$, $f_{s}\left[U_{s}(x, y)\right] \in C\left(\bar{B}_{s}\right), f_{s}: \bar{B}_{s} \rightarrow R, \bar{B}_{s} \subset R^{3}, \Pi p_{x 0 y} \bar{B}_{s}=\bar{D}_{s}$, причому $U_{2}\left(x, y_{1}\right)=U_{1}\left(x, y_{1}\right), x \in\left[x_{0}, x_{1}\right], U_{3}\left(x_{1}, y\right)=U_{1}\left(x_{1}, y\right), y \in\left[y_{0}, y_{1}\right]$.

Зауважимо, що умови (5) є умовами узгодженості крайових умов (2)-(4), а (6) - умови неперервності розв'язку задачі (1)-(5), якщо він існує. Права частина рівняння (1) $f[U(x, y)]$ всюди неперервна функція в області $\bar{B}:=\bar{B}_{1} \cup \bar{B}_{2} \cup \bar{B}_{3}$, за виключенням характеристик $x=x_{1}$, $y=y_{1}$ рівняння (1), вздовж яких вона може мати скінчені розриви. 
Очевидно, якщо існує розв'язок задачі (1)-(5) $U(x, y)$, то $U(x, y)=U_{s}(x, y),(x, y) \in \bar{D}_{s}, s=1,2,3$, де $U_{1}(x, y) \in$ розв'язком задачі Гурса (1), (2), (5) при $(x, y) \in \bar{D}_{1}, U_{2}(x, y)$ - задачі Дарбу (1), (3), (5) і першої з умов (6), $(x, y) \in \bar{D}_{2}$, а $U_{3}(x, y)$ - задачі Дарбу (1), (4), (5) і другої з умов (6) при $(x, y) \in \bar{D}_{3}$.

Надалі вважатимемо, що задані функції

$$
\begin{gathered}
a_{1}(x, y) \in C^{(1.0)}(D), a_{2}(x, y) \in C^{(0.1)}(D), \psi(y) \in C^{1}\left[y_{0}, y_{1}\right], \\
\varphi(x) \in C^{1}\left[x_{0}, x_{1}\right], \omega_{r}(x, y) \in C^{1}\left[x_{r-1}, x_{r}\right], r=1,2,
\end{gathered}
$$

причому

$$
a_{1_{x}}(x, y)=a_{2_{y}}(x, y) .
$$

Лема 1. Якщо $f_{s}\left[U_{s}(x, y)\right] \in C\left(\bar{B}_{s}\right)$ і виконується умова (7), то крайова задача (1)-(5) еквівалентна системі інтегральних рівнянь вигляду

$$
\begin{gathered}
U_{s}(x, y)=\gamma_{s}(x, y)+\varepsilon_{s} T_{1, s} F_{1}\left[U_{1}(\xi, \eta)\right]+T_{s} F_{s}\left[U_{s}(\xi, \eta)\right], \\
(x, y) \in \bar{D}_{s}, \quad s=1,2,3, \quad \varepsilon_{1}=0, \quad \varepsilon_{2}=\varepsilon_{3}=1
\end{gathered}
$$

(тут і надалі використовуватимуться позначення, приведені в [4]).

Лема 2. Нехай виконуються умови леми 1 і крайова задача (1)(5) має розв'язок $U(x, y)$.

Тоді він належатиме просторові функцій $C^{(1.1)}(D) \cap C(\bar{D})$ (буде регулярним), якщо $f[U(x, y)] \in C(\bar{B})$ і $\rho_{1}=\rho_{2}=0$. У супротивному випадку він буде іррегулярним.

Надалі будемо вважати, що функції $F_{s}\left[U_{s}(x, y)\right] \in C_{2}\left(\bar{B}_{s}\right)$, тобто, що вони задовольняють наступні умови $[6$, с. 79$]$ :

1) $F_{s}\left[U_{s}(x, y)\right] \in C\left(\bar{B}_{s}\right)$;

2) в просторі функцій $C\left(\bar{B}_{s, 1}\right), \bar{B}_{s, 1} \subset R^{4}, \Pi p_{x о y} \bar{B}_{s, 1}=\bar{D}_{s}, \quad s=1,2,3$, існує така функція

$$
H_{s}\left[x, y, U_{s}(x, y) ; V_{s}(x, y)\right]:=H_{s}\left[U_{s}(x, y) ; V_{s}(x, y)\right] \text {, що }
$$

(a) $H_{s}\left[U_{s}(x, y) ; V_{s}(x, y)\right] \equiv F_{s}[U(x, y)]$,

(b) для довільної пари неперервних функцій $U_{s}(x, y)$, $V_{s}(x, y) \in \bar{B}_{s, 1}$, які задовольняють умови $U_{s}(x, y) \geq V_{s}(x, y)$, $(x, y) \in \bar{D}_{s}$, в області $\bar{B}_{s, 1}$ виконується нерівність

$$
H_{s}\left[U_{s}(x, y) ; V_{s}(x, y)\right]-H_{s}\left[V_{s}(x, y) ; U_{s}(x, y)\right] \leq 0 ;
$$


3) функція $H_{s}\left[U_{s}(x, y) ; V_{s}(x, y)\right]$ в області $\bar{B}_{s, 1}$ задовольняє умові Ліпшиця, тобто, для довільних двох пар неперервних в $\bar{D}_{s}$ функцій $U_{s, r}(x, y), V_{s, r}(x, y) \in \bar{B}_{s, 1}$, виконується умова

$$
\left|H\left[U_{s, 1}(x, y) ; U_{s, 2}(x, y)\right]-H\left[V_{s, 1}(x, y) ; V_{s, 2}(x, y)\right]\right| \leq L_{s}\left(\left|W_{s, 1}(x, y)\right|+\left|W_{s, 2}(x, y)\right|\right),
$$

де $W_{s, r}(x, y):=U_{s, r}(x, y)-V_{s, r}(x, y), r=1,2$, а $L_{s}$ - стала Ліпшиця, $s=1,2,3$.

Неважко переконатися, що якщо функція $F_{s}\left[U_{s}(x, y)\right] \in \in C\left(\bar{B}_{s}\right)$ і має обмежену частинну похідну першого порядку по $U_{s}(x, y)$, то вона завжди належить просторові $C_{2}\left(\bar{B}_{s}\right), s=1,2,3$. Зворотне твердження не справедливе.

Нехай $z_{s, p}(x, y), v_{s, p}(x, y) \in C\left(\bar{D}_{s}\right) \quad$ належать області $\bar{B}_{s, 1}$ для всіх $s=1,2,3$ та $p \in N_{0}:=N \cup\{0\}$.

Введемо позначення:

$$
\begin{gathered}
f_{s}^{p}(x, y):=H_{s}\left[z_{s, p}(x, y) ; v_{s, p}(x, y)\right], \\
f_{s, p}(x, y):=H_{s}\left[v_{s, p}(x, y) ; z_{s, p}(x, y)\right], \\
R_{s}^{p}(x, y):=\gamma_{s}(x, y)+\varepsilon_{s} T_{1, s} f_{1}^{p}(\xi, \eta)+T_{s} f_{s}^{p}(\xi, \eta), \\
R_{s, p}(x, y):=\gamma_{s}(x, y)+\varepsilon_{s} T_{1, s} f_{1, p}(\xi, \eta)+T_{s} f_{s, p}(\xi, \eta), \\
\alpha_{s, p}(x, y):=z_{s, p}(x, y)-R_{s}^{p}(x, y), \\
\beta_{s, p}(x, y):=v_{s, p}(x, y)-R_{s, p}(x, y), \\
\bar{z}_{s, p}(x, y):=z_{s, p}(x, y)-q_{s, p}(x, y) W_{s, p}(x, y), \quad p \in N, \\
\bar{v}_{s, p}(x, y):=v_{s, p}(x, y)+c_{s, p}(x, y) W_{s, p}(x, y), \\
F_{s}^{p}(x, y):=H\left[\bar{z}_{s, p}(x, y) ; \bar{v}_{s, p}(x, y)\right], \\
F_{s, p}(x, y):=H\left[\bar{v}_{s, p}(x, y) ; \bar{z}_{s, p}(x, y)\right], \\
\bar{R}_{s}^{p}(x, y):=\gamma_{s}(x, y)+\varepsilon_{s} T_{1, s} F_{1}^{p}(\xi, \eta)+T_{s} F_{s}^{p}(\xi, \eta), \\
\bar{R}_{s, p}(x, y):=\gamma_{s}(x, y)+\varepsilon_{s} T_{1, s} F_{1, p}(\xi, \eta)+T_{s} F_{s, p}(\xi, \eta),
\end{gathered}
$$

де $q_{s, p}(x, y), c_{s, p}(x, y)$ є довільними з простору $C\left(\bar{D}_{s}\right)$ функціями, які задовольняють умови

$$
0 \leq q_{s, p}(x, y) \leq 0,5, \quad 0 \leq c_{s, p}(x, y) \leq 0,5,
$$

для всіх $p \in N$ та $(x, y) \in \bar{D}_{s}, s=1,2,3\left(q_{s, 0}(x, y)=c_{s, 0}(x, y)=0\right)$. 
Побудуємо послідовності функцій $\left\{z_{s, p}(x, y)\right\}$ та $\left\{v_{s, p}(x, y)\right\}$ згідно формул [7, с. 132]

$$
z_{s, p+1}(x, y)=\bar{R}_{s}^{p}(x, y), v_{s, p+1}(x, y)=\bar{R}_{s, p}(x, y),
$$

де за нульове наближення $z_{s, 0}(x, y), v_{s, 0}(x, y) \in \bar{B}_{s, 1}$ вибираємо довільні з простору $C\left(\bar{D}_{s}\right)$ функції, які при $(x, y) \in \bar{D}_{s}$ задовольняють умови

$$
W_{s, 0}(x, y) \geq 0, \alpha_{s, 0}(x, y) \geq 0, \beta_{s, 0}(x, y) \leq 0,(x, y) \in \bar{D}_{s} .
$$

Означення. Функції $z_{s, 0}(x, y), v_{s, 0}(x, y) \in C\left(\bar{D}_{s}\right), s=1,2,3$, які належать області $\bar{B}_{s, 1}$ і задовольняють умови (12), називаються функціями порівняння крайової задачі (1)-(5).

Лема 3. Якщо $F_{s}\left[U_{s}(x, y)\right] \in C_{2}\left(\bar{B}_{s}\right)$, то множина функцій порівняння задачі (1)-(5) не порожня.

Лема 4. Якщо $F_{s}\left[U_{s}(x, y)\right] \in C_{2}\left(\bar{B}_{s}\right)$ та інтегральні рівняння (8) в просторі функцій $C\left(\bar{D}_{s}\right), s=1,2,3$ мають розв'язки $U_{s}(x, y)$, які задовольняють нерівності

$$
v_{s, 0}(x, y) \leq U_{s}(x, y) \leq z_{s, 0}(x, y), \quad(x, y) \in \bar{D}_{s}, \quad s=1,2,3,
$$

то $\alpha_{s, 0}(x, y) \geq 0$ і $\beta_{s, 0}(x, y) \leq 0, \quad(x, y) \in \bar{D}_{s}$.

Теорема. Нехай функції $F_{s}\left[U_{s}(x, y)\right] \in C_{2}\left(\bar{B}_{s}\right), \quad$ a $a_{1}(x, y) \in C^{(1.0)}(D), a_{2}(x, y) \in C^{(0.1)}(D)$ і виконуються умови (7).

Тоді послідовності функцій $\left\{z_{s, p}(x, y)\right\}$ та $\left\{v_{s, p}(x, y)\right\}$, побудовані згідно закону (10), (11), (12), де функції $q_{s, p}(x, y), c_{s, p}(x, y)$ на кожному кроці ітерації вибираються таким чином, щоб виконувалися умови

$$
\begin{array}{r}
\bar{R}_{s}^{p}(x, y)-\bar{R}_{s, p-1}(x, y)-c_{s, p}(x, y) W_{s, p}(x, y) \geq(\leq) 0, \\
\bar{R}_{s, p}(x, y)-\bar{R}_{s}^{p-1}(x, y)+q_{s, p}(x, y) W_{s, p}(x, y) \leq(\geq) 0, \\
(x, y) \in \bar{D}_{s}, \quad s=1,2,3 \text { при } p-\text { парних (непарних) i } \\
v_{s, 0}(x, y) \leq z_{s, 1}(x, y), z_{s, 0}(x, y) \geq v_{s, 1}(x, y):
\end{array}
$$

1) збігаються рівномірно до єдиного неперервного розв'язку $U_{s}(x, y)$ відповідного інтегрального рівняння в (8) при $(x, y) \bar{D}_{s}$, $s=1,2,3$;

2) в області $\bar{B}_{s, 1}$ виконуються нерівності 


$$
\begin{gathered}
v_{s, 2 p}(x, y) \leq z_{s, 2 p+1}(x, y) \leq v_{s, 2 p+2}(x, y) \leq z_{s, 2 p+3}(x, y) \leq U_{s}(x, y) \leq \\
\leq v_{s, 2 p+3}(x, y) \leq z_{s, 2 p+2}(x, y) \leq v_{s, 2 p+1}(x, y) \leq z_{s, 2 p}(x, y)
\end{gathered}
$$

для всіх $(x, y) \in \bar{D}_{s}, s=1,2,3$;

3) справедливі оцінки

$$
\max _{s} \sup _{\bar{D}_{s}}\left|W_{s, p}(x, y)\right| \leq \frac{1}{p !}\left[k q \gamma l\left(x-x_{0}+y-y_{0)}\right]^{p} \cdot d,\right.
$$

де

$$
\begin{gathered}
\max _{s} \sup _{\bar{D}_{s}}\left|W_{s, 0}(x, y)\right|=d, \max _{s} L_{s}=l, \\
\max _{s, p} \sup _{\bar{D}_{s}}\left(1-q_{s, p}(x, y)-c_{s, p}(x, y)\right) \leq q, \quad s=1,2,3, \\
\max \left\{1, \sup _{\bar{D}}\left(y-y_{0}+x-x_{0}\right)\right\}=\gamma, \\
\max _{s} \sup _{\bar{D}_{s} \times \bar{D}_{s}}|K(x, y ; \xi, \eta)| \leq 0,5 k ;
\end{gathered}
$$

4) збіжність ітераційного методу (10) - (15) не повільніша збіжності методу $(11)$, коли всі $q_{s, p}(x, y)=c_{s, p}(x, y)=0$ i, отже,

$$
F_{s}^{p}(x, y)=f_{s}^{p}(x, y), \quad F_{s, p}(x, y)=f_{s, p}(x, y) .
$$

Лема 5. Якщо функції $F_{s}\left[U_{s}(x, y)\right] \in C_{2}\left(\bar{B}_{s}\right)$ і виконуються нерівності (15), то множина функцій $q_{s, p}(x, y), c_{s, p}(x, y)$, які задовольняють умови (10), (14) не порожня.

Для доведення леми 5 достатньо покласти

$$
\begin{gathered}
q_{s, p}(x, y)=\left\{\begin{array}{cr}
\left(\beta_{s, p}(x, y)+w_{s, p}(x, y)\right) \cdot \tau_{s, p}^{-1}(x, y), & w_{s, p}(x, y) \neq 0, \\
0, & w_{s, p}(x, y)=0
\end{array}\right. \\
c_{s, p}(x, y)=\left\{\begin{array}{cr}
\left(-\alpha_{s, p}(x, y)+w_{s, p}(x, y)\right) \cdot \tau_{s, p}^{-1}(x, y), & w_{s, p}(x, y) \neq 0, \\
0, & w_{s, p}(x, y)=0
\end{array}\right.
\end{gathered}
$$

де $\tau_{s, p}(x, y):=\alpha_{s, p}(x, y)-\beta_{s, p}(x, y)+w_{s, p}(x, y)$.

Наслідок 1. Нехай виконуються умови теореми. Тоді в просторі функцій $C^{*}(\bar{D})$ існує єдиний іррегулярний розв'язок задачі (1)-(4). Якщо ж права частина рівняння (1) $f[U(x, y)] \in C(\bar{B})$ і виконуються умови $\rho_{\kappa}=0, \kappa=1,2$, то розв'язок крайової задачі (1)-(4) буде регулярним (тобто $\left.U(x, y) \in C^{(1.1)}(D) \cap C(\bar{D})\right)$.

Наслідок 2. Якщо виконуються умови теореми, то нерівності (12) є необхідними і достатніми, щоб виконувались умови (13). 
Наслідок 3. Нехай виконуються умови теореми і $\psi(y)=$

$$
\begin{aligned}
& =\varphi(x)=0, \quad(x, y) \in \bar{D}_{1}, \quad \omega_{r}(x)=0, \quad x \in\left[x_{r-1}, x_{r}\right], \quad r=1,2, \quad \text { a } \\
& F_{s}\left[U_{s}(x, y)\right] \equiv H\left[U_{s}(x, y) ; 0\right] .
\end{aligned}
$$

Тоді, якщо $F_{s}[0] \leq(\geq) 0$ в області $\bar{B}_{s}$, то розв'язок крайової задачі (1)-(4) при $(x, y) \in \bar{D}$ задовольняє нерівності $U(x, y) \leq(\geq) 0$.

\section{Список використаних джерел:}

1. Marynets V. V., Marynets K. V. On Goursat-Darboux boundary-value problem for systems of non-linear differential equations of hyperbolic type. Miskolc Mathematical Notes. 2013. Vol. 14, N 3. P. 1009-1020.

2. Маринець В. В., Маринець К. В., Питьовка О. Ю. Про одну крайову задачу теорії ДРЧП гіперболічного типу в області із складною структурою краю. Наук. Вісник УэнНУ. Сер. матем. і інформ. 2014. 25, № 2. С.110-117.

3. Маринець В. В., Питьовка О. Ю. Про один підхід дослідження крайових задач для нелінійних рівнянь гіперболічного типу в області зі складною структурою краю. Математичне та комп ютерне моделювання. Серія: Фіз.-мат. науки : зб. наук. праць ІК ім. В. М. Глушкова НАНУ. 2017. Вип. 15. С. 113-119.

4. Маринець В. В., Питьовка О. Ю. Дослідження крайової задачі для нелінійного хвильового рівняння з розривною правою частиною. Наук. Вісник УжНУ. Сер. матем. і інформ. 2018. Вип. № 1(32). С. 127-134.

5. Collatz L. Funktionalanalysis und numerische mathematic. Berlin ; Göttingen ; Heidelberg: Springeler-Verlag, 1964. P. 440.

6. Marynets V. V., Marynets K. V., Pytovka O. Yu. On one constructive method of the differential equations of the hyperbolic type. Науковий вісник Ужгородського університету. 2015. Вип. № 2 (27). Р. 76-85.

7. Красносельский М. А., Вайникко Г. М., Забрейко П. П., Рутицкий Я. Б., Стеценко В. Я. Приближенное решение операторных уравнений. М. : Наука, 1969. 456 с.

\section{ON ONE APPROACH OF INVESTIGATION OF THE BOUNDARY VALUE PROBLEM FOR A QUASILINEAR HYPERBOLIC TYPE EQUATION WITH DISCONTINUITIES IN THE RIGHT HAND — SIDE}

We build a constructive method for investigation of the boundary value problem for the wave equation in the domain with complex structure of the boundary.

Key words: "free» curves, irregular solution, comparison functions, consistency conditions.

Одержано 20.01.2019 